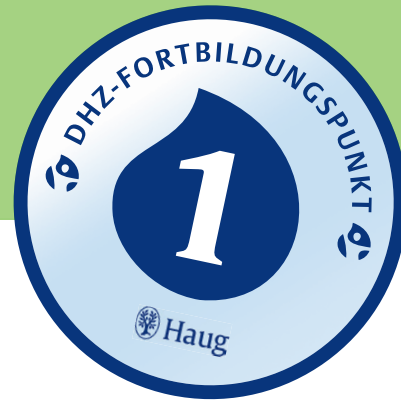

Viele Leser der DHZ sammeln DHZFortbildungspunkte für das freiwillige Fortbildungszertifikat für Heilpraktiker. Seit 2010 unterstützen folgende Fachverbände die FobiPunkte der DHZ als Nachweis für die kontinuierliche Fortbildung an: BDH, AGAHP, BDC, DAGC, HP-Gesellschaft für Elektroakupunktur nach Voll e.V., $\mathrm{HPGO}_{3}$.

Heilpraktiker bilden sich sehr engagiert und regelmäßig fort - ganz ohne Zwang und Pflicht, einfach, weil sie wissen, dass sie dies ihren Patienten schuldig sind und eine Praxis sich heutzutage auch kaum anders erfolgreich führen ließe. Doch tatsächlich sind sie nach der Berufsordnung für Heilpraktiker (BOH, Artikel 5) zur ständigen Fortbildung verpflichtet. Diese Fortbildung müssen sie zudem nachweisen - im Extremfall vor Gericht.

Ganz unabhängig davon ist eine kontinuierliche Qualifizierung heutzutage unerlässlich. Nicht nur Politiker und maßgebliche Vertreter des Gesundheitswesens fordern eine dokumentierte Qualitätssicherung. Moderne, aufgeklärte Patienten erwarten ebenfalls - völlig zu Recht - aktualisiertes Wissen und umfassende Kompetenz auf neuestem Stand von ihren Therapeuten.

\section{Fortbildungszertifikate - längst} Alltag in vielen Medizinberufen

Viele medizinische Berufe haben inzwischen ein eingeführtes Fortbildungsprogramm, das kontinuierliches Weiterlernen fordert, dokumentiert, zertifiziert und nicht zuletzt auch das Ansehen des
Zertifizierten steigert. Das CME-System (continuing medical education) der Ärzte hat sich weltweit durchgesetzt. Aber auch die Apotheker, pharmazeutisch-technische Assistenten, Pflegekräfte, Physiotherapeuten und einige Heilpraktikerverbände haben die Notwendigkeit der kontinuierlichen Fortbildung erkannt und inzwischen ihre eigenen Fortbildungszertifikate entwickelt. Gerade vor dem Hintergrund der Berufsstandssicherung ist der Nachweis von qualifizierter Fortbildung von zentraler Bedeutung. So bietet z. B. der Bund Deutscher Heilpraktiker (BDH) gemeinsam mit AGAHP, BDC, DAGC, HP-Gesellschaft für Elektroakupunktur nach Voll e.V. und $\mathrm{HPGO}_{3}$ ein freiwilliges Fortbildungszertifikat für Heilpraktiker an. Diese Institutionen nutzen die DHZFortbildungspunkte, um ihren Mitgliedern interaktive Fortbildung zu ermöglichen.

\section{DHZ-Punkte sammeln \\ für das Zertifikat}

Das freiwillige Fortbildungszertifikat für Heilpraktiker der genannten Fachverbände basiert im Wesentlichen auf dem oben beschriebenen generell anerkannten Fortbildungsmodell (CME) und garantiert so die Akzeptanz durch Öffentlichkeit, andere Gesundheitsberufe und die Politik. Die Grundeinheit dieser Fortbildungsaktivitäten ist der Fortbildungspunkt (FP). Dieser entspricht i. d. R. einer abgeschlossenen Fortbildungsstunde (45 min). Für die erworbenen Fortbildungspunkte muss ein Nachweis erbracht werden. Punkte können z. B. entweder durch den Besuch von Fortbildungsveranstaltungen, Kongressen, Fachtagungen oder Workshops gesammelt oder über sog. interaktive Fachfortbildungen durch Internet oder Fachzeitschriften erworben werden. Bei der interaktiven Fachfortbildung sind der Nachweis der Qualifizierung und die schriftliche Auswertung des Lernerfolgs eine Grundvoraussetzung für die Vergabe der Fortbildungspunkte.

Das Fortbildungszertifikat für Heilpraktiker wird ausgestellt, wenn Sie in 3 Jahren 120 Fortbildungspunkte erworben, diese dokumentiert und einen entsprechenden Antrag auf Zertifizierung bei der Dokumentationsstelle des BDH gestellt haben. Davon können Sie maximal 10 Punkte pro Jahr für das Zertifikat mithilfe der interaktiven Fortbildung durch Fachzeitschriften sammeln (Infos unter www.bdh-online.de).

Das Fortbildungszertifikat steht allen Heilpraktikern offen, unabhängig von ihrer Verbandszugehörigkeit.

\section{Homöopathie-Zertifikat: Punkte erwerben mit der DHZ}

Die SHZ ist eine gemeinnützige, von Einzelinteressen unabhängige Stiftung des bürgerlichen Rechts.

Sie hat die Aufgabe, ein hohes Qualitätsniveau in der Aus- und Weiterbildung von Homöopathen (Heilpraktikern und Ärzten) zu fördern und zu sichern, mit dem Ziel, Qualitätskriterien für eine klassisch homöopathische Behandlung in der Öffentlichkeit zu etablieren. Die umfassenden Qualifikationsrichtlinien definieren Inhalt und Umfang homöopathischer Ausbildungen (mind. 550 Unterrichtseinheiten Kontaktstudium und 1250 Unter- 


\section{DHZ-Fortbildungspunkte - Fragebogen}

Es ist jeweils nur eine Aussage pro

Auswahl korrekt. Bitte wählen Sie bei allen Aussagen die jeweils richtige. Die Aussagen beziehen sich auf die Artikel S. 14-21 in der Rubrik Spektrum.

\section{Welche Aussage trifft jeweils zu?}

\section{Auswahl 1}

a Die Pathophysiognomik nach Natale Ferronato ist eine Ausschlussdiagnose.

b Die Pathophysiognomik nach Natale Ferronato ist eine Hinweisdiagnose.

c Bei der Pathophysiognomik nach Natale Ferronato zeigen bestimmte Fußareale organische Schwächen sowie aktuelle und frühere Belastungen.

d Bei der Pathophysiognomik nach Natale Ferronato sind Farbveränderungen der Gesichtshaut nicht von Bedeutung.

e Geschminkte Haut kann die Hinweisdiagnostik nach Natale Ferronato erheblich vereinfachen.

\section{Auswahl 2}

a Um Veränderungen wahrnehmen zu können, stellt Natale Ferronato als Normstruktur und Normfarbe der Haut bestimmte Kriterien auf.

b Eine Mischfarbe im Wangenbereich kann ein Hinweis auf Multiple Sklerose sein.

c Veränderungen der Feinstruktur der Gesichtshaut zeigen sich immer an mehreren Stellen der Hautzonen.

d Man unterscheidet ausschließlich zwischen den Hautqualitäten trocken und fettig.

e Eine Schwellung der Hautzone deutet meist auf eine Stauung in den dieser Hautzone zugeordneten Organen hin.

\section{Auswahl 3}

a Das Organsystem des Magens zeigt sich an den Augenbrauen.

b Das Organsystem des Magens zeigt sich am Kinn.

c Das Organsystem des Magens zeigt sich an den Mundwinkeln.

d Linksseitig der Mundwinkel liegt die Hautreflexzone für die kleine Krümmung des Magens.

e Rechtsseitig der Mundwinkel liegt die Hautreflexzone für die große Krümmung des Magens.

\section{Auswahl 4}

a Schwellungen deuten auf eine Trägheit des Magens hin.

b Einziehungen weisen auf eine Mangelernährung hin. c Eine gelbe Verfärbung im Bereich der Magenreflexzone weist auf eine Gastritis hin.

d Eine rote Verfärbung im Bereich der Magenreflexzone weist auf Helicobacter pylori hin.

e Eine vom Mundwinkel ausgehende gerade nach unten führende Falte weist auf einen entzündlichen Prozess hin.

\section{Auswahl 5}

a Erscheint die Färbung der Oberlippe wie orange gepudert, ist dies ein Hinweis auf eine Verminderung der Resorptionsfähigkeit der Nahrungsbestandteile.

b Die Nasolabialfalte entspricht der Anatomie der Leber.

c Die rechte Nasolabialfalte präsentiert die linke Seite des Herzens.

d Die Beschaffenheit der Nasolabialfalte (im Querschnitte gesehen) hat physiologisch eine klare V-Form über die gesamte Länge der Falte.

e Abweichungen von der V-Form erzählen von einer Überforderung des Magens.

\section{Auswahl 6}

a Die Café-au-lait-Haut kann Hinweis sein auf eine Vitamin-B-Mangel-Anämie, es sollte daher z. B. auch auf Schleimhautdefekte oder Subikterus geachtet werden.

b Die kalkweiße Blässe tritt bei Niereninsuffizienz auf, es sollte auch auf Symptome wie Uhrglasnägel oder Zyanose geachtet werden.

c Eine grau-zyanotische Blässe beobachtet man meist bei Hämolyse oder Leberschaden.

d Subikterus beobachtet man meist bei Hämolyse oder Leberschaden. Weitere Symptome sind Haarausfall und Hypermenorrhö.

e Ödeme, Pruritus und Foetor ex ore sind Kardinalsymptome der Rechtsherzinsuffizienz.

\section{Auswahl 7}

a Eine plötzlich einsetzende, einseitige Rötung ist typisch für das sog. Mitralgesicht.

b Bei einer Scharlachinfektion kommt es zu einem Erysipel im Gesicht, meist um das Mund-Nasen-Dreieck (sog. Drosselbart).

c Anzeichen der Rosazea ist eine sich langsam entwickelnde, entzündliche Rötung. Sie findet sich ausschließlich im Gesicht (beidseitig).

d Beim Cushing-Syndrom ist eine über das gesamte Gesicht ausgebreitete Rötung typisch, Ursache ist der Hypokortisolismus.

e Bei der Polyglobulie kommt es zu einer umschriebenen Rötung an einer Seite des Halses. Der Patient hat meist hohes Fieber.

\section{Auswahl 8}

a Ein kardiales Ödem findet sich v. a. bei einer Rechtsherzinsuffizienz oder ungestauter Linksherzinsuffizienz.

b Ödeme bei einer Hyperalbuminämie treten generalisiert auf, z. B. bei Niereninsuffizienz oder Malabsorptionssyndrom.

c Ein Myxödem findet sich bei einer Schilddrüsenfunktionsstörung. Bei einer Hyperthyreose ist es prätibial lokalisiert, insbesondere beim Morbus Basedow.

d Lipödeme sind Proteineinlagerungen, oft mit konsekutivem Lymphstau, die sich hauptsächlich bei Männern finden.

e Das allergische Ödem zeigt sich mehrere Stunden nach Kontakt mit einem Allergen als diffuse Rötung.

\section{Auswahl 9}

a Ölfilmnägel zeigen sich in einer orangebraunen, diffus umschriebenen oder scharf abgegrenzten Verfärbung der Nagelplatte und sind ein typisches Zeichen der Eisenmangelanämie.

b Uhrglasnägel sind große, konvex gewölbte Nägel, die bei Erkrankungen mit chronischer Hypoxie wie Herz- und/oder Lungenerkrankungen sowie Leberzirrhose vorkommen.

c Weiße Flecken in der Nagelplatte sind Zeichen der Erschöpfungszyanose.

d Als Palmarerythem bezeichnet man eine diffuse Rötung der Handaußenflächen, meist unter Aussparung der zentralen Tiefen.

e Tophi sind typisch für akute Gichtanfälle, die Hände sind rot, aufgedunsen und überwärmt.

\section{Auswahl 10}

a Der schwankende, torkelnde Gang mit überschießenden unkoordinierten Bewegungen (Seemannsgang) ist immer physiologisch, er kommt nach Schiffsreisen vor.

b Der Scherengang fällt durch Adduktion mit Überkreuzen der Beine (lange, ausladende Schritte mit Drehung des Körpers auf dem Standbein) auf. Typisch bei Adduktorenspasmus und Diplegie.

c Beim Rigor fallen die stockenden, eingeschränkten, wenig geschmeidigen, motorisch meist überschießenden Bewegungen auf. Typisch bei Morbus Parkinson und depressiver Verstimmung.

d Bei Patienten, die nach vorne gebeugt sitzen, sollte an eine Behinderung/ Einschränkung der Atmung (Asthma bronchiale) gedacht werden.

e Sehr stark aufgerichtete Patienten leiden meist unter Schmerzen im unteren LWS-Bereich, ein Bandscheibenvorfall muss unbedingt ausgeschlossen werden. 


\section{DHZ-Fortbildungspunkte - Antwortbogen}

\section{DHZ-Antworten}

Lernerfolgskontrolle.

Bitte nur eine Antwort pro Auswahl ankreuzen! Sie können in dieser DHZ-Ausgabe 2 Fortbildungspunkte erzielen.

$\begin{array}{lllllll}\text { Frage } & 1 & \text { a } & \text { b } & \text { C } & \text { d } & \text { e } \\ \text { Frage } & 2 & \text { a } & \text { b } & \text { C } & \text { d } & \text { e } \\ \text { Frage } & 3 & \text { a } & \text { b } & \text { c } & \text { d } & \text { e } \\ \text { Frage } & 4 & \text { a } & \text { b } & \text { C } & \text { d } & \text { e } \\ \text { Frage } & 5 & \text { a } & \text { b } & \text { c } & \text { d } & \text { e }\end{array}$

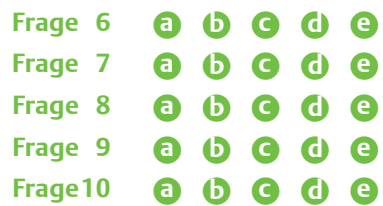

\section{Angaben zur Person}

\section{Feld für DHZ-Wertmarke|Abo-Nummer}

Nicht-Abonnenten bitte hier DHZ-Wertmarke aufkleben. Abonnenten bitte Abonummer eintragen.

\section{Erklärung}

Ich versichere, dass ich die Beant-

wortung der Fragen selbst und ohne Hilfe durchgeführt habe.
Ort | Datum
Unterschrift

\section{Ihr Ergebnis}

$\square$ Sie haben

Fragen richtig beantwortet.
Sie haben

DHZ-Punkte erworben.
Sie haben nicht erfolgreich teilgenommen.

Einsendeschluss 27.06.2013 (Datum des Poststempels)

Bitte senden Sie den vollständigen Antwortbogen zusammen mit einem

an Sie selbst adressierten und ausreichend frankierten Rückumschlag an den 
richtseinheiten Heimstudium). Sie stellen sicher, dass die überprüften Therapeuten die klassische Homöopathie in Theorie und Praxis beherrschen. Für Homöopathen nach der Ausbildung ist neben einer Zertifikatsprüfung eine 3-jährige Supervisionszeit obligatorisch. Mit dem Erhalt des Zertifikats verpflichten sich die zertifizierten Homöopathen, ihr Wissen ständig auf dem Laufenden zu halten. Regelmäßige Fortbildungen auf homöopathischem (30 Unterrichtseinheiten à 45 min pro Jahr) und schulmedizinischem Gebiet (8 Unterrichtseinheiten à 45 min pro Jahr) müssen alle 2 Jahre nachgewiesen werden, um die Zertifizierung aufrechtzuerhalten (Infos unter www.homoeopathie-zertifikat.de).

Die SHZ hat sich seit der Ausgabe DHZ 6/2008 entschieden, allen Zertifikatsinhabern die Chance der interaktiven Fortbildung anzubieten, um den Nachweis für die klinische Fortbildung zu erbringen. So können die Zertifikatsinhaber die Fortbildungspunkte der DHZ bei der SHZ als Nachweis für die klinische Fortbildung einreichen (max. 4 Unterrichtseinheiten pro Jahr - das entspricht 4 Fortbildungspunkten der DHZ).

\section{Verbånde und unabhångige Stiftung stehen für Qualitåt}

Das Entscheidende an diesen beiden Systemen ist, dass Teilnehmer nicht einfach Teilnahmebescheinigungen von Fortbildungen sammeln können, sondern dass eine Zertifizierungsstelle, hier ein Berufsverband bzw. eine Stiftung, für die Qualität der Fortbildung steht und diese überprüft und dokumentiert.

Das DHZ-Team unterstützt den Ansatz der regelmäßigen qualifizierten und dokumentierten Fortbildung. Die interaktive Fortbildung halten wir für ganz besonders geeignet, eine qualifizierte Fortbildung zu belegen, weil hier eine eindeutige Lernerfolgskontrolle besteht. Nur wer die Fragen richtig beantwortet, erhält auch seine Fortbildungspunkte.

\section{So geht's}

Sie finden in jeder DHZ-Ausgabe Fortbildungseinheiten. Diese können Sie - auch unabhängig von der Zertifizierung - regelmäßig nutzen, um Ihren Lernerfolg zu überprüfen und zu dokumentieren. Andererseits können Sie sich diese DHZ-Fortbildungs- punkte auch für die Fortbildungszertifikate der genannten Verbände anerkennen lassen.

\section{Anerkennung der DHZ-Beitråge}

Die Vergabe der Fortbildungspunkte kann direkt durch die DHZ erfolgen.

Pro Lerneinheit erhalten Sie 2 Fortbildungspunkte. Hierfür

- müssen 7 von 10 Fragen richtig beantwortet sein

- muss der Antwortbogen vollständig ausgefüllt sein

- muss im entsprechenden Feld des Antwortbogens eine DHZ-Wertmarke aufgeklebt oder Ihre DHZ-Abo-

Nummer eingetragen sein

Die Abo-Nummer finden Sie auf dem Adressaufkleber Ihres Heftes

\section{Beantworten und abschicken!}

$\mathrm{Zu}$ einzelnen Beiträgen der DHZ gibt es in jeder Ausgabe Fragebögen (S. II), die Sie ab 2010 auch im Internet unter www.thiemeconnect.de/ejournals/toc/dhz (auch als Nicht-Abonnent der DHZ) herunterladen und ausdrucken können. Schicken Sie uns einfach den ausgefüllten Antwortbogen (S. III). Wir prüfen Ihre Antworten und benachrichtigen Sie über Ihren Lernerfolg. Die Bescheinigung können Sie dann bei der zentralen Dokumentationsstelle des Fortbildungszertifikats für Heilpraktiker beim BDH und für das Homöopathiezertifikat bei der SHZ einreichen.

\section{Die Teilnahme ist rückwirkend bis} max. 1 Jahr nach Erscheinen der jeweiligen DHZ-Ausgabe möglich. Beachten Sie den Einsendeschluss auf dem Antwortbogen.

\section{Datenschutz}

Ihre Daten werden ausschließlich für die Bearbeitung dieser Fortbildungseinheit verwendet. Es erfolgt beim Verlag keine Speicherung der Ergebnisse über die für die Bearbeitung der Fortbildungseinheit notwendige Zeit hinaus. Die Daten werden nach Versand der Punktbescheinigungen anonymisiert. Namen- und Adressangaben auf den Antwortbögen dienen ausschließlich dem Versand der Punktbescheinigungen. Die Angaben zur Person dienen nur statistischen Zwecken und werden von den Adressangaben getrennt und anonymisiert verarbeitet.

\section{DHZ-Wertmarke für}

\section{Nicht-Abonnenten}

DHZ-Wertmarken können beim Verlag erworben werden: 6er-Pack DHZ-Wertmarken; Preis 25,00 € inkl. MwSt.; Artikel-Nr. 902999

\section{Bitte richten Sie Ihre Bestellung an: \\ MVS Medizinverlage Stuttgart \\ KundenServiceCenter Buch \\ Postfach 301120; 70451 Stuttgart \\ Tel.: 0711/8931-900}

Dieser Artikel ist online zu finden unter: http://dx.doi.org//10.1055/s-0032-1321950

\section{Teilnahme}

Jeder Heilpraktiker soll die Möglichkeit zur Teilnahme an der Fortbildung erhalten. Deshalb ist die Teilnahme an der DHZ-Fortbildung nicht an ein Abonnement geknüpft. Dennoch sollten Sie im Besitz einer $\mathrm{DHZ}$ sein, denn alle im Fragebogen enthaltenen Fragen beziehen sich auf die Spektrumbeiträge der jeweiligen Ausgabe. An diversen Heilpraktikerschulen finden Sie DHZExemplare, die dort ausliegen. Darüber hinaus können Sie als Nicht-Abonnent der DHZ Einzelexemplare erwerben bei: KundenServiceCenter Zeitschriften, Rüdigerstr. 14, 70469 Stuttgart, Tel.: 0711/8931-308. Für Abonnenten ist die Teilnahme kostenfrei. Nicht-Abonnenten können DHZ-Wertmarken erwerben. 